\title{
Assistência de enfermagem à mulher no climatério e menopausa: estratégia de inclusão na rotina das unidades básicas de saúde
}

A contagem da menopausa é realizada a partir da última ocorrência da menstruação até os 12 meses seguintes. Neste período as mulheres apresentam alterações hormonais como: falta de desejo sexual e de libido, calores e sudorese excessiva e baixa autoestima, cefaleia, menstruações irregulares e com maior fluxo, caracterizando, assim, o climatério. O presente estudo tem como objetivo expor o valor do enfermeiro no atendimento à população feminina no climatério e menopausa. Para tal, foi feita uma pesquisa descritiva, quantitativa e qualitativa, tendo como principal fonte de pesquisa uma revisão de literatura. Foram utilizados trabalhos científicos em português, inglês e espanhol sendo selecionados trinta artigos já publicados entre os anos de 2015 a 2020 , cujas bases de dados foram LILACS, SCIELO, MEDILINE utilizando os seguintes descritores: climatério; menopausa. Embora seja um assunto muito falado, o climatério e menopausa é um tabu na sociedade, onde as mulheres desconhecem os sinais e sintomas, tratamento e como melhorar sua saúde. Desta maneira, é sugerido o debate do assunto no eixo acadêmico, como a implantações dos programas para a socialização entre mulheres na mesma faixa etária, o incentivo de troca de experiência através de roda de conversas, e o desenvolvimento das políticas públicas que incentivem a atenção primaria a oferecerem um atendimento individualizado e holístico as essas mulheres. Sendo assim, o que grande parte dos estudos analisados nesse trabalho traz são reflexões quanto à importância da assistência de enfermagem a mulher no período do climatério e menopausa.

Palavras-chave: Climatério; Menopausa; Enfermagem; Saúde da mulher.

\section{Nursing assistance to women in the climate and menopause: an inclusion strategy in the route of the basic health units}

Menopause counting is carried out from the last occurrence of menstruation until the next 12 months. In this period, women have hormonal changes such as: lack of sexual desire and libido, excessive heat and sweating and low self-esteem, headache, irregular menstruation and with greater flow, thus characterizing the climacteric. The present study aims to expose the value of nurses in assisting the female population in menopause and menopause. To this end, a descriptive, quantitative and qualitative research was carried out, with the main source of research being a literature review. Scientific papers were used in Portuguese, English and Spanish, with thirty articles already published between the years 2015 to 2020 being selected, whose databases were LILACS, SCIELO, MEDILINE using the following descriptors: climacteric; menopause. Although it is a widely talked about subject, menopause and menopause is a taboo in society, where women are unaware of the signs and symptoms, treatment and how to improve their health. In this way, it is suggested the debate of the subject in the academic axis, such as the implantation of programs for socialization among women in the same age group, the incentive to exchange experiences through round of conversations, and the development of public policies that encourage attention primary care to offer individualized and holistic care to these women. Therefore, what most of the studies analyzed in this work brings are reflections on the importance of nursing care for women during the climacteric and menopause periods.

Keywords: Climacteric; Menopause; Nursing; Women's health.

Bruna Aguiar Sabóia

Faculdade Guaraí, Brasil

http://lattes.cnpq.br/6589749401480274

brunaaguiar@hotmail.com

\section{Mayza Carla Silva Rosa}

Faculdade Guaraí, Brasil

http://lattes.cnpq.br/3202428704497150

mayzacarla@hotmail.com

Giullia Bianca Ferraciolli do Couto

Faculdade Guaraí, Brasil

http://lattes.cnpq.br/0109560699727614

giulliabianca@hotmail.com
Adriana Keila Dias

Faculdade Guaraí, Brasil

http://lattes.cnpq.br/2128882976477548

adrianakeiladias@hotmail.com

\section{Glaucya Wanderley Santos Markus}

Faculdade Guaraí, Brasil

http://lattes.cnpq.br/5568510365985231

glaucyamarkus@outlook.com

Juliane Marcelino dos Santos

Faculdade Guaraí, Brasil

http://lattes.cnpq.br/8751832120632358

julianemarcelino@hotmail.com
Reobbe Aguiar Pereira

Faculdade Guaraí, Brasil

http://lattes.cnpq.br/7447115724350334

enfreobbe@gmail.com

Karla Camila Correia da Silva

Faculdade Guaraí, Brasil

http://lattes.cnpq.br/1981447087125364

karlacamilac@yahoo.com.br d

DOI: 10.6008/CBPC2236-9600.2021.003.0011
Referencing this:

SABÓIA, B. A.; ROSA, M. C. S.; COUTO, G. B. F.; DIAS, A. K. D.; MARKUS, G. W. S.; SANTOS, J. M. S.; PEREIRA, R. A.; SILVA, K. C. C.. Assistência de enfermagem à mulher no climatério e menopausa: estratégia de inclusão na rotina das unidades básicas de saúde. Scire Salutis, v.11, n.3, p.80-89, 2021. DOI: http://doi.org/10.6008/CBPC2236$\underline{9600.2021 .003 .0011}$ 


\section{INTRODUÇÃO}

De acordo com o Instituto Brasileiro de Geografia e Estatística (IBGE), em 2010, o número de mulheres entre 60 e 79 anos, no estado do Tocantins era de 9.631 .724 e no município de Guaraí, região central do estado apresentou um total de 901 mulheres entre 60 e 79 anos de vida, essas mulheres atravessam o período do climatério e menopausa (IBGE, 2020). Em consequência do crescimento da expectativa de vida e aumento da população na terceira idade, estima-se que até 2030 cerca de 2 milhões e meio de mulheres alcançarão o climatério e a menopausa.

A Organização mundial da saúde, caracteriza o climatério como uma fase biológica na vida da mulher, onde ocorre a transição do final da fase reprodutiva para não reprodutiva. De modo que a menopausa é caracterizada como a parada da menstruação feminina. (Caderno de atenção mulher no climatério) protocolo de atenção básica, 2016. A contagem da menopausa é realizada a partir da última ocorrência da menstruação até os 12 meses seguintes. Neste período as mulheres apresentam alterações hormonais como: falta de desejo sexual e de libido, calores e sudorese excessiva e baixa autoestima, cefaleia, menstruações irregulares e com maior fluxo, caracterizando, assim, o climatério (BRASIL, 2020).

Dentro das inúmeras práticas realizadas pelo enfermeiro, a consulta de enfermagem vem sendo uma ferramenta de grande valia, pois será o momento onde este poderá identifica as queixas através de uma boa anamnese, orientando, implementando e desenvolvendo um plano de cuidado a está mulher, incluindo orientação quanto a qualidade de vida e hábitos saudáveis, além de, tranquiliza-la quanto aos sinais e sintomas que poderão aparecer ao logo do processo do climatério. Diante disto, o presente estudo tem como objetivo expor o valor do enfermeiro no atendimento à população feminina no climatério e menopausa. Tendo como objetivos específicos, o conceituar a menopausa e o climatério; levantar na literatura especializada as principais mudanças no corpo no metabolismo da mulher durante a menopausa; identificar a atuação da enfermagem na assistência à mulher durante o processo da menopausa e climatério.

\section{METODOLOGIA}

O presente artigo é uma pesquisa descritiva, quantitativa e qualitativa, tendo como principal fonte de pesquisa uma revisão de literatura. A pesquisa bibliográfica foi elaborada com base em material já escrito, constituído em artigos científicos e caderno de atenção básica da mulher e protocolos da saúde. Para a construção deste estudo, as informações necessárias foram buscadas através diversos trabalhos científicos em português, inglês e espanhol sendo selecionados trinta artigos já publicados entre os anos de 2015 a 2020, cujas bases de dados foram LILACS, SCIELO, MEDILINE utilizando os seguintes descritores: climatério; menopausa;

Os artigos apontados pela estratégia de busca foram avaliados de forma autônomas, pelo pesquisador (autor), obedecendo exatamente aos critérios de inclusão: texto de forma integra, tempo da busca, sendo a população-alvo (mulheres), as intervenções no (climatério), e idioma (português, inglês e espanhol). Foram excluídos os estudos que não obedeceram aos critérios de inclusão citados acima. 
Os dados teóricos foram discutidos à principal temática dos autores por meio de análise interpretativa sobre o tema do estudo, o climatério e menopausa. A figura 01 apresenta um fluxograma que representa a busca, seleção e análise dos artigos utilizados para o presente trabalho.

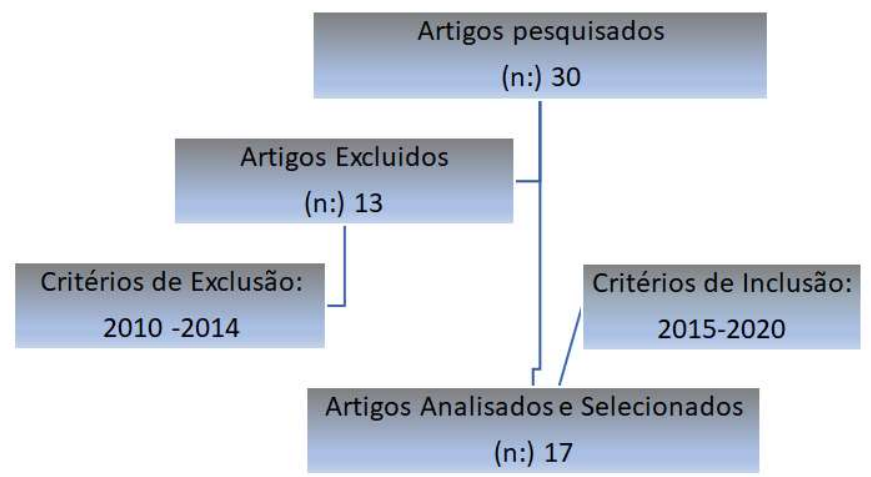

Figura 1: Fluxograma representativo da busca, seleção e análise dos trabalhos analisados

\section{DISCUSSÃO TEÓRICA}

O envelhecimento da população feminina está em aumento constante, estudos apontam que cerca de 30 milhões de mulheres estão nesta faixa etária de 35 A 65, o que cresceu a expectativa de vida para entorno de 74 anos. Durante todo o desenvolvimento feminino estas mulheres passam por uma grande evolução: nascer, entra na puberdade onde ocorre a primeira menarca, passa pelo aperto de ciclos menstruais irregulares ou regulares, gravidez, e por último climatério e menopausa (CALDAS et al., 2015).

A menarca é o nome da primeira menstruação, ela ocorre entre 10 aos 15 anos, variando de acordo com cada organismo. Nesta fase ocorre o desenvolvimento das mamas, aparecimento dos pelos pubianos e desenvolvimento dos hormônios estrogênio e progesterona que auxiliam na produção dos ovários. A menina, se torna mulher, ocorre uma carga emocional, onde a alteração hormonal e corporal trazem ansiedade e estresse ao dia a dia, gerando expectativa de como viver a demais fase que sucederam a menarca (BRASIL, 2020). A Menacme é um período onde a mulher entra no período fértil, ele está presente entre a puberdade e a menopausa. Neste período muitas, iniciam a atividade sexual, onde ocorre o rompimento do hímen. Uma fase de descoberta na vida da mulher, onde ela já tem autonomia sobre o corpo, encontra-se sexualmente ativa e já tem liberdade de escolher seu parceiro (BRASIL, 2020)

O climatério marca o período onde a mulher passa pelo cessamento do período reprodutivo. Algumas literaturas relatam que esta fase tem surgindo mais cedo, na faixa etária dos 35 anos iniciando assim os primeiros sintomas emocional, vasomotores e etc. Isso ocorre devido à queda hormonal, hábitos alimentares irregulares, falta de atividade física e isolamento social (ARANHA et al., 2016). A menopausa também tem começado a surgir mais cedo, através dos períodos entre os 40 a 50 anos. Neste período a população feminina tem uma carência de informação e de acolhimento, muitas se isolam e sofrem os sinais e sintomas sem ter uma orientação adequada. De forma mais aberta, abordaremos estas fases do climatério e menopausa (LEITE et al., 2020).

A fase pré menopausa é caracterizada pelo desaparecimento de menstruação com duração de 
aproximadamente 3 meses (FIGUEIREDO JUNIOR et al., 2020). A fase perimenopausa iniciam-se as irregularidades menstrual com duração aproximadamente de 3 a 11 meses, isso ocorre devido ao esgotamento ovariano, reduzindo a produção do hormônio estrógeno a aumenta o LH e o FHS; alteração psicológicas e sexuais. Determinados por sintomas como: onda de calores, irritabilidade, insônia, falha na memória, mudança repentina no humor, ocorre ressecamento de pele, mucosa, cabelo, atrofia e estreitamento do canal vaginal (CALDAS et al., 2015). A fase pós-menopausa é um marco natural na vida da mulher, nela ocorre a parada da menstruação, onde nas literaturas ela se apresenta após 12 meses sem a decida do fluxo sanguíneo (SOUZA et al., 2015).

A menopausa traz a estas mulheres muitas mudanças, de forma mais explicativa alguns autores dividem os sinais e sintomas em curto, médio e longo prazo, isso varia de acordo com o comportamento corporal. O curto prazo, a chegada da menopausa pode causar muito calor, alteração no humor, como depressão ou irritação, dor de cabeça e diminuição do desejo sexual. Em médio prazo, caracteriza-se como baixa procura por sexo, ocorre atrofia e ressecamento da mucosa vaginal, dor na penetração. Em longo prazo, pode ocorrer o surgimento de osteoporose, doenças cardiovasculares, pois o estrogênio hormônios sexuais femininos, tem como principal função de protege o coração, veias e pequenos vasos sanguíneos, causando diminuição da proteção e queda do hormônio. Neste estágio as mulheres tendem a desenvolver patologias ósseas e cardiovasculares (BRASIL, 2020).

As mulheres neste período não compreendem o momento em qual estão passando, os sinais sintomas são muito confusos, ficam nervosas com a família, sentem vergonha, não conseguem resolver seus dilemas. Sendo assim, o climatério tem uma influência negativa na vida das mulheres que passam por este período, mesmo que achemos que o mundo está em crescente desenvolvimento, este assunto ainda é muito tabu no meio feminino, o que ocasiona uma desmotivação na busca pela qualidade de vida, abalando o emocional, o trabalho e a convivência familiar (PEIXOTO et al., 2020). Outra dificuldade encontrada é a vivencia da sexualidade, com a alteração dos hormônios estas mulheres tendem a não conhecer as alterações no corpo, tais como as atrofias sexuais, secura vaginal, e acabam criando conflitos com seus parceiros e perdem o interesse pela sexualidade (BRASIL, 2020).

Diante do exposto, as mulheres ouvem falar da menopausa porem, muitas mulheres desconhecem as mudanças de corpo. Nesta fase do climatério muitas mulheres começam a sentir os sintomas apresentados no período da menopausa, se descuidam dos métodos contraceptivos, acreditando que já findaram sua vida reprodutiva, o que muitas vezes ocasiona uma gravidez indesejada, por não terem conhecimento (PATRICIO et al., 2020).

\section{Agravos à saúde durante o período do climatério}

A menopausa e o climatério estão presentes em partes na vida das mulheres, e ocasionam mudanças metabólicas que podem trazer agravamentos como hipotireoidismo, doenças cardiovasculares, hipertensão arterial, obesidade, diabetes e osteoporose (FEBRASGO, 2020).

O hipotiroidismo é a deficiência da produção de hormônios na glândula da tireoide. Os sintomas 
não são específicos, porem esses sintomas se apresentam na menopausa, algum desses sintomas também são: queda de cabelo, ressecamento da pele, aumento ou perda de peso repentino, metabolismo lento. $O$ hipotireoidismo pode ser descoberto através de exames laboratoriais como o TSH E T4 livre (BRASIL, 2020).

A cardiopatia é uma patologia que se manifestada no coração. As mulheres após os 50 anos estão propensas ao risco de infarto e acidente vascular cerebral (AVC), devido a mudança hormonal na menopausa, apresentam também alterações circulatória e sanguínea, hipertensão arterial, diabetes e falta de atividade física. Com a diminuição do estrogênio no climatério a mulher está susceptível a tromboses, aumento do colesterol, principalmente o LDL, com presença da perda da proteção do HDL (PATRICIO et al., 2020).

A Obesidade pode aparecer em qualquer fase da vida, porem na menopausa ela afeta as mulheres que estão em mudança de metabolismo. Com o estresse e ansiedade gerada neste período, ocorre o excesso de consumo de calorias a mais do que a quantidade diária necessária ao corpo, e com a falta de motivação, essas mulheres não realizam atividade física, com isso ocorre o aparecimento de placas de gorduras presentes em todo o corpo. Sendo assim, a pessoa obesa está mais propensa a desenvolver doenças neoplásicas e coronarianas (GONÇALVES et al., 2015).

A Diabetes é uma patologia crônica causada pela deficiência da insulina ou quando o corpo não consegue absorver a insulina. Sabemos que a insulina é um hormônio que controla a quantidade de glicose no organismo, e é dividida em dois tipos: A tipo 1 é caracterizada como a deficiência de insulina, sendo que o paciente será insulina dependente. Já a tipo dois é caracterizada como resistente a insulina e disfunção da célula beta, este paciente poderá fazer uso ou não de medicamento, isso vai depender da avaliação do médico. Segundo estudos, esta patologia se apresenta na menopausa, devido ao envelhecimento e alteração hormonal, o que compromete o dia a dia destas mulheres. A prevenção é a chave de auxilio neste envelhecimento, onde a mulher desde a infância deverá ter uma alimentação adequada e praticar exercício físico diário (GARCIA et al., 2020).

A osteoporose é uma doença descrita como diminuição da massa óssea e da perda da microarquitetura do tecido ósseo, causando grande risco de fraturas e fragilidade óssea, e tem seu surgimento na pós menopausa, na faixa etária entre 50 a 60 anos dependendo de cada organismo. Tem o desenvolvimento em decorrência de uma desordem do processo de remodelação óssea, onde o esqueleto de um indivíduo em fase adulta é composto de osso cortical (compacto) e trabécula (esponjoso), é constantemente é reformado e reparado pela remodelação óssea, onde este processo é importante para a manutenção do esqueleto. $O$ envelhecimento, causam a mulher um desgaste na remodelação óssea onde passam a apresentar fragilidade. Sendo assim as mulheres na menopausa, estão expostas ao risco de queda e fratura, devido a lentidão do metabolismo na remodelação óssea (FEBRASGO, 2020).

\section{Tratamentos Indicados no climatério}

O climatério e menopausa tem começado cada vez mais cedo na vida das mulheres, trazendo muitas dúvidas, incertezas, medos e principalmente sinais e sintomas. Como forma de auxilio, o ministério 
da Saúde desenvolveu manuais sobre o climatério e menopausa, e dentro destes documentos se fala das práticas da terapia hormonal, terapias medicamentosas não hormonais, medicamentos fitoterápicos e acupuntura. Estas práticas tem crescido no brasil e ajuda as mulheres a passarem por esse processo e a amenizar os sinais e sintomas nesta fase natural da vida feminina.

\section{Terapia hormonal}

O tratamento da Terapia Hormonal (TH) visa diminuir os sintomas vasomotores, a secura vaginal para evitar a dispaurenia, melhora do sono e aumentar a libido. Segundo o Ministério da Saúde, a TH deve ser começada com o objetivo de aliviar os sintomas que tanto incomodam as mulheres, que ocorrem pela diminuição dos esteroides, tais como sudorese, alterações menstruais. O quadro 01 abaixo mostra os medicamentos utilizados durante a TH.

\begin{tabular}{|l|c|c|c|}
\hline \multirow{2}{*}{} & \multicolumn{3}{|c|}{ Dose } \\
\cline { 2 - 4 } & Baixa & Tradicional & Alta \\
\hline Estrogênios conjugados (oral) & 0,3 ou $0,45 \mathrm{mg}$ & $0,625 \mathrm{mg}$ & $1,25 \mathrm{mg}$ \\
\hline Estradiol (oral) & $0,5 \mathrm{mg}^{*} / 1 \mathrm{mg}^{* *}$ & $1 \mathrm{mg}^{*} / 2 \mathrm{mg}^{* *}$ & $2 \mathrm{mg}^{*} / 4 \mathrm{mg}^{* *}$ \\
\hline Estradiol transdérmico (adesivo) & $25 \mathrm{mcg}$ & $50 \mathrm{mcg}$ & $100 \mathrm{mcg}$ \\
\hline Estradiol percutâneo (gel) & $0,5 \mathrm{mg}-0,75 \mathrm{mg}$ & $1 \mathrm{mg}-1,5 \mathrm{mg}$ & $2 \mathrm{mg}$ \\
\hline
\end{tabular}

Quadro 1: Medicamentos usados na Terapia Hormonal (TH). Fonte: BRASIL (2020)

No tratamento é contraindicado quando as pacientes apresentam histórico de tromboembolia aguda e repetitivo; câncer de mama e endométrio; sangramento genital sem identificação da causa e doença hepática grave A Diabetes Melittus não controlada, a hipertensão arterial, endometriose e miomatose uterina, nestes casos o profissional medico irá analisar estas pacientes para só depois prescrever o tratamento ou descarta-lo.

\section{Tratamento medicamentoso não hormonal}

\begin{tabular}{|l|l|l|l|}
\hline $\begin{array}{c}\text { Classe de } \\
\text { Medicamentos }\end{array}$ & \multicolumn{1}{|c|}{ Fármaco } & \multicolumn{1}{c|}{ Dose } & \multicolumn{1}{c|}{ Mecanismo de Ação } \\
\hline \multirow{2}{*}{$\begin{array}{l}\text { Antidopaminér- } \\
\text { gicos }\end{array}$} & Veraliprida & $100 \mathrm{mg} /$ dia & Ação central \\
\cline { 2 - 5 } & Sulpiride & $100 \mathrm{mg} /$ dia & Ação central \\
\cline { 2 - 4 } & $\begin{array}{l}\text { Domperi- } \\
\text { done }\end{array}$ & $10 \mathrm{a} 20 \mathrm{mg} /$ dia & Ação periférica \\
\hline \multirow{2}{*}{ Antidepressivos } & Venlafaxina & $\begin{array}{l}37,5 \mathrm{mg} \text { a } 75 \mathrm{mg} / \\
\text { dia }\end{array}$ & $\begin{array}{l}\text { Ação central - inibidores da recap- } \\
\text { tação da serotonina e noradrenalina }\end{array}$ \\
\hline \multirow{2}{*}{$\begin{array}{l}\text { Antidepressivos } \\
\text { tricíclicos }\end{array}$} & $\begin{array}{l}\text { Carbonato } \\
\text { de lítio }\end{array}$ & $300 \mathrm{mg} /$ dia & Ação central \\
\cline { 2 - 5 } & Imipramina & $25 \mathrm{a} 50 \mathrm{mg} /$ dia & Ação central \\
\cline { 2 - 5 } & Nomifensina & $25 \mathrm{a} 50 \mathrm{mg} /$ dia. & Ação central \\
\hline $\begin{array}{l}\text { Antidepressivos } \\
\text { tetracíclicos }\end{array}$ & $\begin{array}{l}\text { Cloridrato } \\
\text { de fluoxetina }\end{array}$ & $20 \mathrm{mg} /$ dia & Ação central \\
\hline
\end{tabular}

Quadro 2: Tratamento medicamentos não hormonal. Fonte: (BRASIL, 2020).

O tratamento dos sinais e sintomas presentes no climatério e menopausa também podem ser por medicamentos não hormonal. Este tratamento é usado para melhoria dos sintomas vasomotores, tais como: onda de calores e sudorese, somente é usado quando classificados como raro ou equilibrado. As indicações terapêuticas são: mulheres que sofrem com os efeitos colaterais, contra indicação para a TH; para mulheres que não querem fazer o uso de hormonioterapia; para mulheres com sintomatologia ao resultado da terapia hormonal insatisfatória. As opções disponíveis para a terapêutica são os agentes 
antidopaminérgicos, antidepressivos, hipno-sedativos, vasoativos e os que atuam no eixo hipotalâmico hipofisário que estão descritos nos quadros 02 e 03. Estes medicamentos descritos nos quadros 2 e 3 auxiliam na melhoria dos sinais e sintomas vasomotores (fogachos e sudorese), nas mudanças de humor, insônia.

\begin{tabular}{|c|c|c|c|}
\hline $\begin{array}{c}\text { Classe de } \\
\text { Medicamentos }\end{array}$ & Fármaco & Dose & Mecanismo de Açâo \\
\hline \multirow{2}{*}{ Hipno-sedativos } & Fenobarbital & $50 \mathrm{mg} / \mathrm{dia}$ & $\begin{array}{l}\text { Agem no metabolismo do ácido } \\
\text { gama-aminobutírico }\end{array}$ \\
\hline & $\begin{array}{l}\begin{array}{l}\text { Alfametil- } \\
\text { dopa }\end{array} \\
\end{array}$ & $\begin{array}{l}250 \mathrm{mg} \text { a } \\
500 \mathrm{mg} / \text { dia }\end{array}$ & Ação inibidora das catecolaminas \\
\hline \multirow{6}{*}{ Vasoativos } & Benciclano & $300 \mathrm{mg} / \mathrm{dia}$ & $\begin{array}{l}\text { Ação vasodilatadora cerebral e } \\
\text { periférica }\end{array}$ \\
\hline & Cinarizina & $75 \mathrm{mg} / \mathrm{dia}$ & $\begin{array}{l}\text { Açào anti-histamínica, age por } \\
\text { competição H } 1\end{array}$ \\
\hline & Clonidina & 0,1 a $0,2 \mathrm{mg} / \mathrm{dia}$ & $\begin{array}{l}\text { Açào hipotensora, agonista alfa- } \\
\text { adrenérgico }\end{array}$ \\
\hline & \begin{tabular}{|l|} 
Derivadas do \\
Esporão do \\
Centeio \\
\end{tabular} & 4,5 a $20 \mathrm{mg} / \mathrm{dia}$ & Ação vasodilatadora \\
\hline & Nicergolina & $300 \mathrm{mg} /$ dia & Ativadora do metabolismo cerebral \\
\hline & Propanolol & $80 \mathrm{mg} /$ dia & Açao beta-bloqueadora \\
\hline \multirow{2}{*}{$\begin{array}{l}\text { Atuam no eixo } \\
\text { hipotalâmico- } \\
\text { hipofisário }\end{array}$} & $\begin{array}{l}\text { Bromoergo- } \\
\text { criptina }\end{array}$ & 1,25 a $2,5 \mathrm{mg} / \mathrm{dia}$ & Deprime os pulsos de $\mathrm{LH}$ \\
\hline & Ciclofenil & 200 a $400 \mathrm{mg} / \mathrm{dia}$ & Açao na reduçào do FSH e Prolactina \\
\hline
\end{tabular}

Quadro 3: Tratamento medicamentos não hormonal. Fonte: BRASIL (2020).

\section{Medicina natural e práticas complementares}

As práticas complementares e a medicina natural foram incluídas na atenção primaria através da Política Nacional de Praticas Integrativas e Complementares (PNPIC) na rede SUS para auxiliar a equipe de saúde nos atendimentos a população. Com a necessidade de incluir mais medicamentos a população, e com a deficiência na demanda de compras de medicamentos para auxiliar a população, o Ministério da Saúde implantou a Política Nacional de Plantas Medicinais e Fitoterápicos e o Programa Nacional de Plantas Medicinais Fitoterápico, com o intuito de aumentar a distribuição medicamentosa.

A fitoterapia é uma terapêutica que utiliza os extratos das plantas medicinais para a composição de medicamentos fitoterápicos. Esta terapia é utilizada há décadas, em forma de ervas e chás, passada de geração a geração. Estes medicamentos auxiliam na melhora dos sinais e sintomas, são usados os fitohormônios que são extraídos das plantas, e são vendidos em forma de capsulas ou comprimidos. Alguns exemplos de medicamentos fitoterápicos como o Trifolium pratense, Glycine max, e a Cimicífuga racemosa são usados como repositores hormonais. Os sintomas psicoemocionais são tratados com os medicamentos fitoterápicos hiperico perforatum, Valeriana officinalis e Melissa officinalis.

A Cimicífuga racenosa é utilizada para reduzir os fogachos; Dioscorea vilosa reduz os sintomas da menopausa; Hypericum perforatum tratam os sintomas da depressão. O profissional para prescrever esta técnica, tem que passar por um treinamento para poder aprender sobre os medicamentos.

Quadro 4: Distribuição dos medicamentos fitoterápicos, classe, identificação, dosagem, contra indicação e efeitos colaterais.

\begin{tabular}{|l|l|l|l|l|}
\hline Classe & Extrato/ indicação & Dosagem & Contraindicação & Efeito colaterais \\
\hline (Glycine max) & $\begin{array}{l}\text { Extrato padronizado de } 40 \% \text { a } \\
70 \% \text { de isoflavonas. }\end{array}$ & $\begin{array}{l}50 \text { a } 180 \mathrm{mg} \text { por } \\
\text { dia, que devem } \\
\text { ssados como repositor hormonal. } \\
\end{array}$ & $\begin{array}{l}\text { Não possuem contraindicação. } \\
\text { duas tomadas } \\
(12 / 12 \mathrm{~h}) .\end{array}$ & $\begin{array}{l}\text { Possíveis efeitos colaterais: } \\
\text { alergias, interferência com a } \\
\text { absorção de certos minerais (Pela } \\
\text { presença de ácido fítico), } \\
\text { constipação, flatulência, náuseas } \\
\text { e irritação gástrica. }\end{array}$ \\
\hline
\end{tabular}




\begin{tabular}{|c|c|c|c|c|}
\hline $\begin{array}{l}\text { Trevo } \\
\text { Vermelho } \\
\text { (Trifolium } \\
\text { pratense) }\end{array}$ & $\begin{array}{l}\text { Trevo vermelho - extrato } \\
\text { padronizado a } 8 \% \text { de isoflavonas. } \\
\text { Alivio dos fogachos. }\end{array}$ & $\begin{array}{l}\text { Uso: } 40 \mathrm{mg} \text { a } \\
60 \mathrm{mg} \text { por dia } \\
\text { com dose única } \\
\text { diária. }\end{array}$ & $\begin{array}{l}\text { Hipersensibilidade aos } \\
\text { componentes da fórmula e } \\
\text { presença de coagulopatias. } \\
\text { Mulheres gestantes ou } \\
\text { amamentando não devem fazer } \\
\text { uso. Em caso de manipulação } \\
\text { cirúrgica de médio e grande porte } \\
\text { - interromper o uso } 48 \text { horas } \\
\text { antes do procedimento. }\end{array}$ & $\begin{array}{l}\text { Semelhantes aos de produtos à } \\
\text { base de isoflavonas. O uso } \\
\text { concomitante de anticoagulantes } \\
\text { orais ou heparina pode ter seu } \\
\text { efeito potencializado. O uso de } \\
\text { contraceptivos hormonais, bem } \\
\text { como de tamoxifem podem } \\
\text { sofrer interferência. } \\
\text { Drogas de metabolização } \\
\text { hepática como antialérgicos } \\
\text { (fexofenadine), antifúngicos } \\
\text { (itraconazol, cetoconazol) } \\
\text { antineoplásicos (paclitaxel, } \\
\text { vimblastina, vincristina) e } \\
\text { redutores de colesterol } \\
\text { (sinvastatina, lovastatina), podem } \\
\text { ter sua ação alterada. }\end{array}$ \\
\hline $\begin{array}{l}\text { Cimicífuga } \\
\text { racemosa }\end{array}$ & $\begin{array}{l}\text { Extrato padronizado entre } 2,5 \text { e } \\
8 \% \text { de } 27 \text {-deoxiacteína } \\
\text { Melhora a atrofia vaginal }\end{array}$ & $\begin{array}{l}\text { Uso: } 40 \text { a } \\
\text { 80mg/dia. Pode } \\
\text { ser associada às } \\
\text { isoflavonas. }\end{array}$ & & $\begin{array}{l}\text { Possíveis efeitos colaterais: são } \\
\text { muito raros. Incluem dor } \\
\text { abdominal, diarreia, cefaleia, } \\
\text { vertigens, náusea, vômito e dores } \\
\text { articulares. }\end{array}$ \\
\hline $\begin{array}{l}\text { Valeriana } \\
\text { (Valeriana } \\
\text { officinalis) }\end{array}$ & $\begin{array}{l}\text { Extrato seco com } 0,8 \% \text { de ácidos } \\
\text { valerênicos. } \\
\text { Ansiedade e insônia. }\end{array}$ & $\begin{array}{l}\text { Uso: } 300 \text { a } \\
400 \mathrm{mg} \text { ao dia, } \\
\text { divididos em } \\
\text { duas a três } \\
\text { tomadas }\end{array}$ & $\begin{array}{l}\text { Hipersensibilidade, gestação e } \\
\text { lactação. }\end{array}$ & $\begin{array}{l}\text { Hipersensibilidade aos } \\
\text { componentes da fórmula. Devem } \\
\text { ser respeitadas as dosagens, pois } \\
\text { em excesso pode causar cefaleia } \\
\text { e agitação. Grandes quantidades } \\
\text { podem induzir a sonhos, } \\
\text { dispepsia e reações alérgicas } \\
\text { cutâneas }\end{array}$ \\
\hline $\begin{array}{l}\text { Melissa } \\
\text { (Melissa } \\
\text { officinalis) }\end{array}$ & $\begin{array}{l}\text { Extrato seco: Não menos que } \\
0,5 \% \text { de óleo volátil contendo } \\
\text { citral; não menos que } 6 \% \text { de } \\
\text { derivados hidroxicinâmicos totais, } \\
\text { calculados como ácido } \\
\text { rosmarínico. Alívio de ansiedade, } \\
\text { insônia e algumas desordens } \\
\text { digestivas como cólicas } \\
\text { intestinais, flatulência, dispepsia. }\end{array}$ & $\begin{array}{l}\text { Uso: } 80 \text { a } 240 \mathrm{mg} \\
\text { ao dia, em três } \\
\text { tomadas }\end{array}$ & $\begin{array}{l}\text { Gestantes, portadores de } \\
\text { glaucoma e de hipertireoidismo e } \\
\text { hipersensibilidade aos } \\
\text { constituintes da planta. }\end{array}$ & $\begin{array}{l}\text { Entorpecimento e bradicardia em } \\
\text { indivíduos sensíveis }\end{array}$ \\
\hline $\begin{array}{l}\text { Hipérico } \\
\text { (Hiperico } \\
\text { perforatum) }\end{array}$ & $\begin{array}{l}\text { Extrato padronizado a } 0,3 \% \text { de } \\
\text { hipericinas equilíbrio emocional e } \\
\text { ao humor. }\end{array}$ & $\begin{array}{l}\text { Uso: } 300 \text { a } 900 \\
\text { mg ao dia. No } \\
\text { caso de utilizar a } \\
\text { maior dose ( } 900 \\
\text { mg), dividir em } 3 \\
\text { tomadas diárias. }\end{array}$ & $\begin{array}{l}\text { Contraindicações: gravidez, } \\
\text { lactação. Evitar exposição ao sol. }\end{array}$ & $\begin{array}{l}\text { Possíveis efeitos colaterais: } \\
\text { Irritação gástrica, sensibilização } \\
\text { cutânea - fotodermatite, insônia, } \\
\text { ansiedade. }\end{array}$ \\
\hline
\end{tabular}

Fonte: BRASIL (2020).

\section{Medicina tradicional chinesa - Acupuntura}

A medicina tradicional chinesa-acupuntura (MTC) originou-se na china, utilizando a linguagem simbólica das leis da natureza, caracterizadas como as cinco energias presentes no corpo, tais como: terra, fogo, agua, metal e madeira, é fundamentada como a teoria do Ying-Yang, divididas no mundo a fora como duas forças ou princípios complementares com opostos diferente, tendo como objetivo equilibrar a duabilidade.

A acupuntura é uma tecnologia na área da saúde que aborda o processo saúde doença no indivíduo, sendo usada sozinha ou acompanhada por outra técnica. É um procedimento que realiza estímulos em locais anatômicos, onde é feito a inserção de agulhas filiformes metálica para a recuperação da saúde ou manutenção da saúde do ser humano. O estimulo de zonas neurorreativas ou ponto de acupuntura, geram promoção de analgesia. Esta técnica proporciona alivio das cefaleias, lombalgias, estresse, insônia, exerce melhora no sistema digestivo, regulação e emagrecimentos relacionados ao funcionamento metabólico, que proporciona as mulheres alivio nos sintomas da menopausa. 


\section{Assistência de enfermagem}

O Ministério da saúde criou o Manual de atenção a mulher no climatério e menopausa como forma de auxiliar os profissionais enfermeiros no atendimento à mulher no climatério e menopausa. O manual apresenta as diretrizes a todos os profissionais na saúde, sendo que neste documento ele borda as competências que o enfermeiro deverá fazer para atender este público, como a consulta de enfermagem (BRASIL, 2020). A enfermagem vem se destacando no atendimento as mulheres, especialmente na saúde pública, tendo sua importância no atendimento à mulher no climatério e menopausa, tem como objetivo, reconhecer os fatores psicológicos, patológicos e fisiológicos no período do climatério durante as consultas de enfermagem (BRASIL, 2020).

A consulta é um momento único a esta mulher, no período do climatério se sente sozinha, confusa, vulnerável, sem conhecimento prévio dos sinais e sintomas. O climatério e menopausa, apesar de ser um tema muito divulgado na sociedade, continua sendo um tabu se falar sobre o assunto, pois estas mulheres não sabem sobre como passarem por esse processo, desconhecem os sintomas vasomotores, agravos que podem aparecer e não conhecem sobre o tratamento para alívio dos sinais e sintomas (SILVA et al., 2020).

O enfermeiro desempenha seu papel de educador, auxiliando esta mulher perceber a naturalidade de entrar nesta fase. Também é importante que o enfermeiro faça uma boa anamnese, coletando o máximo de dados, verificar se ela possui alguma predisposição agravos relacionado a menopausa, orientar sobre métodos contraceptivo, alimentação e ingesta de água, importância da prática de atividade física, solicitar exames e principalmente transmiti segurança ao paciente (SILVA, 2019). O enfermeiro mostrara a esta paciente que é possível enfrentar esta fase do climatério e menopausa, tratando os sinais e sintomas para melhorar a qualidade de vida, mostrando que envelhecer pode ser saudável e prazeroso.

\section{CONCLUSÕES}

No Brasil tem crescido o número de mulheres no climatério e menopausa com estimativa de 300 milhões, sendo que a faixa etária é de 35 a 65 anos. O climatério é um período em que a mulher passa por alterações hormonais, físicas, psicológicas e sexuais, gerando medos e incertezas. Este período é dividido por fases, onde o profissional irá identificar os sinais e sintomas apresentados nestas fases, como sudorese, fogachos, atrofias do canal vaginal, uretral entre outros sintomas. Ocorrem também o esgotamento ovariano, reduzindo a produção do hormônio estrógeno a aumenta o LH e o FHS; já o cessamento do fluxo sanguíneo só ocorrera após os 12 meses sem menstruação. Os agravos na saúde feminina também aparecem neste período, algumas destas patologias são: osteoporose, cardiopatia diabetes, entre outros (CALDAS et al., 2015). Embora seja um assunto muito falado, o climatério e menopausa é um tabu na sociedade, onde as mulheres desconhecem os sinais e sintomas, tratamento e como melhorar sua saúde. Demonstrando assim, a importância do papel da enfermagem à mulher, tendo competência e autonomia para atuar nas consultas de enfermagem. Este contato deverá trazer conforto, conhecimento, auxílio e incentivo a mulher para que tenha melhor qualidade de vida.

Desta maneira, sugerimos o debate do assunto no eixo acadêmico, como a implantações dos 
programas para a socialização desta mulher com outras mulheres na mesma faixa etária, o incentivo de troca de experiência através de roda de conversas, e o desenvolvimento das políticas públicas que incentivem a atenção primaria a oferecerem um atendimento individualizado e holístico as essas mulheres. De um modo geral, grande parte dos estudos descritos nesse estudo, traz no seu bojo, resultados e avaliações que levam as reflexões quanto a importância da assistência de enfermagem a mulher no período do climatério.

\section{REFERÊNCIAS}

ARANHA, J. S.; LIMA, C. B.; LIMA, M. N. F. A.; NOBRE, J. O. C.. Climatério e menopausa: percepção de mulheres usuárias da estratégia saúde da família. Temas de Saúde, v.16, n.2, 2016.

BRASIL. Ministério da Saúde. Manual de atenção à mulher no climatério: menopausa: saúde das mulheres. Brasília: Ministério da Saúde, 2020.

BRASIL. Ministério da Saúde. Protocolos da atenção básica: saúde das mulheres. Brasília: Ministério da Saúde, 2016.

CALDAS, A. J. M.; SILVA, C. M. M.; AQUINO, D. M. C.; ANJOS, F. V.; VIEIRA, I. O.. Vivenciando o climatério: aspectos socioeconômicos, físicos e emocionais. Revista Enfermagem Brasil, 2015.

FIGUEIREDO, J. C. J.; MORAES, F. V.; RIBEIRO, W. A.; PEREIRA, G. L. F. L.; FELICIO, F. C.; ANDRADE, D. L. B.. A influência dos sintomas climatérios na saúde da mulher. Revista Nursing, 2020.

GARCIA, M. C. R.; TELLO, A. A.; LEÓN, A. R.. Factores que influyen en el comportamento de adherencia del paciente com Diabetes Mellitus tipo 2. Villahermosa, v.18, n.3, 2020.

GONÇALVES, J. T. T.; SILVEIRA, M. F.; CAMPOS, M. C. C.; COSTA, L. H. R.. Sobrepeso e obesidade e fatores associados ao climatério. Revista Ciência \& Saúde Coletiva, v.21, n.4, 2015.
LEITE, T. A. S.; NUNES, J. S. S.; PEREIRA, A. J. L.; SILVA, M. L.. Conhecimento de mulheres jovens sobre a menopausa e sintomas climatérios. Brazilian Journal of health Review, v.3, n.3, p.7204-7212, 2020.

PATRICIO, R. S. O.; JÚNIOR, O. C. R.; FERREIRA, S. M. S.; ARAÚJO, T. S.; BRASIL, L. C.; SILVA, J. M.; BARBOSA, M. S.; CORDEIRO, A. V. S.; PEREIRA, L. S.; ARAÚJO, M. H. N.. Ações de enfermagem na promoção da saúde e qualidade de vida de mulheres no climatério. Revista Eletrônica Acervo Enfermagem, v.4, 2020.

PEIXOTO, R. C. A.; TOLENTINO, T. S.; SILVA, W.; FERREIRA, A. F.; CÉSAR, E. S. R.; ALVES, E. R. P.. Climatério: sintomatologia vivenciada por mulheres atendidas na atenção primária. Revista de ciências da saúde nova esperança, v.18, n.1, 2020.

SILVA, A. P. A. A.; PONTES, L. S.. Assistência de Enfermagem às Mulheres no Climatério. Monografia (Graduação em Enfermagem) - Centro Universitário Planalto Central Apparecido dos Santos, Gama, 2020.

SILVA, M. F.. Importância da consulta de enfermagem ginecológica às mulheres no climatério. Anápolis, 2019.

SOUZA, N. L. S. A.; ARAúJO, C. L. O.. Marco do envelhecimento feminino, a menopausa: sua vivência em uma revisão de literatura. Revista Kairós Gerontologia, v.18, n.2, p.149-165, 2015.

A CBPC - Companhia Brasileira de Produção Científica (CNPJ: 11.221.422/0001-03) detém os direitos materiais desta publicação. Os direitos referem-se à publicação do trabalho em qualquer parte do mundo, incluindo os direitos às renovações, expansões e disseminações da contribuição, bem como outros direitos subsidiários. Todos os trabalhos publicados eletronicamente poderão posteriormente ser publicados em coletâneas impressas sob coordenação da Sustenere Publishing, da Companhia Brasileira de Produção Científica e seus parceiros autorizados. Os (as) autores (as) preservam os direitos autorais, mas não têm permissão para a publicação da contribuição em outro meio, impresso ou digital, em português ou em tradução. 